\title{
Posterior Glenoid Wear in Total Shoulder Arthroplasty: Eccentric Anterior Reaming Is Superior to Posterior Augment
}

\author{
Tim Wang MD, Geoffrey D. Abrams MD, Anthony W. Behn MS, \\ Derek Lindsey MS, Nicholas Giori MD, Emilie V. Cheung MD
}

Received: 5 March 2015/Accepted: 24 July 2015/Published online: 5 August 2015

(C) The Association of Bone and Joint Surgeons (B) 2015

\begin{abstract}
Background Uncorrected glenoid retroversion during total shoulder arthroplasty may lead to an increased likelihood of glenoid prosthetic loosening. Augmented glenoid components seek to correct retroversion to address posterior glenoid bone loss, but few biomechanical studies have evaluated their performance.

Questions/purposes We compared the use of augmented glenoid components with eccentric reaming with standard glenoid components in a posterior glenoid wear model. The primary outcome for biomechanical stability in this model
\end{abstract}

The institution of one or more of the authors (GDA, EVC, NG) has received, during the study period, funding from the Office of Research and Development (Rehabilitation R\&D Service), Department of Veteran Affairs.

One of the authors certifies that he or she (EVC), or a member of his or her immediate family, has or may receive payments or benefits, during the study period, an amount of less than USD 10,000 from Exactech Inc (Gainesville, FL, USA).

One of the authors certifies that he or she (GDA) has or may receive payments or benefits, during the study period, an amount of less than USD 10,000 from in Cytonics Inc (Jupiter, FL, USA).

All ICMJE Conflict of Interest Forms for authors and Clinical Orthopaedics and Related Research ${ }^{\circledR}$ editors and board members are on file with the publication and can be viewed on request. Clinical Orthopaedics and Related Research ${ }^{\mathbb{R}}$ neither advocates nor endorses the use of any treatment, drug, or device. Readers are encouraged to always seek additional information, including FDAapproval status, of any drug or device prior to clinical use. This study was performed at Veterans Affairs Palo Alto (implant preparation) and at Stanford Orthopaedic Surgery Laboratory (biomechanical testing and analysis).

T. Wang ( $\bowtie)$, G. D. Abrams, A. W. Behn, E. V. Cheung Department of Orthopaedic Surgery, Stanford University, 450 Broadway Street, Pavilion C, 4th Floor, Redwood City, CA 94063, USA

e-mail:wangtim@stanford.edu; wang.tim@gmail.com was assessed by (1) implant edge displacement in superior and inferior edge loading at intervals up to 100,000 cycles, with secondary outcomes including (2) implant edge load during superior and inferior translation at intervals up to 100,000 cycles, and (3) incidence of glenoid fracture during implant preparation and after cyclic loading.

Methods A $12^{\circ}$-posterior glenoid defect was created in 12 composite scapulae, and the specimens were divided in two equal groups. In the posterior augment group, glenoid version was corrected to $8^{\circ}$ and an $8^{\circ}$-augmented polyethylene glenoid component was placed. In the eccentric reaming group, anterior glenoid reaming was performed to neutral version and a standard polyethylene glenoid component was placed. Specimens were cyclically loaded in the superoinferior direction to 100,000 cycles. Superior and inferior glenoid edge displacements were recorded.

Results Surviving specimens in the posterior augment group showed greater displacement than the eccentric reaming group of superior $(1.01 \pm 0.02[95 \% \mathrm{CI}$, $0.89-1.13$ ] versus $0.83 \pm 0.10$ [95\% CI, 0.72-0.94 mm]; mean difference, $0.18 \mathrm{~mm} ; \mathrm{p}=0.025$ ) and inferior markers $(1.36 \pm 0.05$ [95\% CI, 1.24-1.48] versus $1.20 \pm 0.09$ [95\% $\mathrm{CI}, 1.09-1.32 \mathrm{~mm}$; mean difference, $0.16 \mathrm{~mm} ; \mathrm{p}=0.038$ ) during superior edge loading and greater displacement of the superior marker during inferior edge loading (1.44 \pm 0.06 [95\% CI, 1.28-1.59] versus $1.16 \pm 0.11$ [95\% CI, $1.02-1.30 \mathrm{~mm}$; mean difference, $0.28 \mathrm{~mm} ; \mathrm{p}=0.009$ ) at 100,000 cycles. No difference was seen with the inferior marker during inferior edge loading $(0.93 \pm 0.15$ [95\% CI,

G. D. Abrams, D. Lindsey, N. Giori

Center for Tissue Regeneration, Repair, and Restoration,

Veterans Affairs Palo Alto Health Care System, Palo Alto, CA, USA 
0.56-1.29] versus $0.78 \pm 0.06$ [95\% CI, $0.70-0.85 \mathrm{~mm}$ ]; mean difference, $0.15 \mathrm{~mm} ; \mathrm{p}=0.079)$. No differences in implant edge load were seen during superior and inferior loading. There were no instances of glenoid vault fracture in either group during implant preparation; however, a greater number of specimens in the eccentric reaming group were able to achieve the final 100,000 time without catastrophic fracture than those in the posterior augment group.

Conclusions When addressing posterior glenoid wear in surrogate scapula models, use of angle-backed augmented glenoid components results in accelerated implant loosening compared with neutral-version glenoid after eccentric reaming, as shown by increased implant edge displacement at analogous times.

Clinical Relevance Angle-backed components may introduce shear stress and potentially compromise stability. Additional in vitro and comparative long-term clinical followup studies are needed to further evaluate this component design.

\section{Introduction}

The number of shoulder arthroplasties performed has increased $250 \%$ during the last 10 years, with nearly 27,000 procedures performed in the United States in 2008 $[1,12,25]$. Primary glenohumeral osteoarthritis is the most common indication for total shoulder arthroplasty, accounting for $77 \%$ of cases [25]. Walch et al. [40] reported $41 \%$ of patients with glenohumeral arthritis have preoperative posterior glenoid wear or posterior subluxation of the humeral head.

Late radiographic lucency and clinical loosening of the glenoid component have been critical concerns in longterm implant survivorship in total shoulder arthroplasty $[15,18,27,36]$. In a review of nearly 3000 total shoulder arthroplasties, Bohsali et al. [5] reported the incidence of aseptic loosening to be $39 \%$ after 5 years, with $83 \%$ of cases attributed to failure of the glenoid component. Shoulder arthroplasty in the setting of posterior glenoid bone loss is associated with a threefold increase in stress in the cement mantle and sevenfold increase in glenoid component micromotion [13, 14, 37]. Glenoid component retroversion decreases glenohumeral contact area, increases contact pressure, and may lead to eccentric loading with resultant glenoid component loosening [14, 37].

Despite its frequency, there is disagreement regarding how to best address posterior glenoid bone loss during total shoulder arthroplasty, and treatment guidelines have not been clearly established $[19,22]$. Some surgeons do not [24] correct mild peripheral bone deficiencies and accept a more-retroverted orientation of the glenoid implant. For larger defects, eccentric anterior reaming and posterior bone graft augmentation are two frequently used techniques, performed either alone or in combination [36]. However, eccentric reaming of the anterior glenoid is limited by the amount of bone that can be removed safely without risking glenoid vault perforation and is recommended only for retroversion less than $15^{\circ}[10,16]$. Additionally, eccentric anterior reaming results in medialization of the glenohumeral joint line and thereby may reduce the surface area of supportive bone where the glenoid prosthesis sits. Posterior corticocancellous bone graft is another option for treating larger posterior glenoid deficiencies but remains a technically challenging procedure with variable outcomes [21, 39]. Although it allows for preservation of glenoid bone stock and restoration of the anatomic joint line, complications such as graft loosening, subsidence, and resorption have been observed in $18 \%$ to $30 \%$ of cases [22].

Posterior-augmented glenoid components have been introduced to compensate for posterior glenoid deficiency. Although proponents advocate the ability to restore the native joint line, few biomechanical or clinical studies have evaluated the performance of these components [22]. An anatomic study [35] showed that these augmented glenoid components may decrease the amount of glenoid vault medialization necessary and more accurately correct glenoid retroversion. Clinical series using augmented glenoid components often are limited by sample size and followup duration, [31, 35], with results of persistent glenohumeral instability and increased risk of failure [9].

Although prior biomechanical studies of augmented glenoid components have been completed, one was performed with synthetic testing materials (nonanatomic models) and nonstandardized testing protocols [23]. Iannotti et al. [23] used a surrogate scapula model to define the biomechanical stability of augmented glenoid components compared with eccentric reaming with standard glenoid components in a posterior glenoid deficiency model, taking into account glenoid anatomy, version, and glenoid plane medialization. Implant fixation and stability with time between groups was assessed by comparing the primary outcome of implant edge displacement in superior and inferior edge loading at intervals up to 100,000 cycles, with a secondary outcome of evaluating implant edge load during superior and inferior translation. We also asked whether eccentric reaming of the anterior glenoid leads to increased incidence of glenoid vault fracture during implant preparation or after cyclic loading. We hypothesized that an angle-backed posterior-augmented glenoid component, when subjected to cyclic loading, would show increased edge displacement than eccentric reaming with placement of a standard glenoid prosthesis. Additionally, we predicted use of an augmented implant would lead to 
decreased edge load and lower likelihood of cortical perforation during preparation.

\section{Materials and Methods}

Twelve composite scapulae were obtained for biomechanical testing (Fourth Generation Sawbones Scapula, Part \# 3413; Pacific Research Laboratories, Vashon, WA, USA). These models are composed of an outer synthetic cortical shell with inner cancellous bone analogue designed to simulate the mechanical properties of bone. A K-wire was driven through the center of the articular surface using a drill guide manufactured with a $12^{\circ}$-posterior angle referenced from the glenoid face [32]. A cannulated reamer was used in line with the guidewire to create a posterior glenoid defect at a $12^{\circ}$-angle in all specimens [11]. Reaming was performed to the same anatomic level among all specimens and was stopped before removal of anterior glenoid rim bone to maintain a consistent amount of substrate.

In the posterior augment group, version of the glenoid face was corrected to $8^{\circ}$ posterior wear and an $8^{\circ}$-allpolyethylene pegged angle-backed posterior-augmented glenoid component was cemented in place according to the manufacturer's protocol (Equinoxe ${ }^{\circledR}$ Shoulder System; Exactech Inc, Gainesville, FL, USA). In the eccentric reaming group, eccentric reaming of the anterior glenoid was performed to create a neutral-version glenoid and a standard pegged all-polyethylene glenoid component was cemented according to the manufacturer's protocol (Exactech Inc). Both glenoid components have identical material properties and similar radius of curvature with three staggered pegs perpendicular to the articular surface of the implant (Fig. 1). All implants were manufactured from GUR 1050 polyethylene and sterilized in inert packaging via gamma irradiation to nominally $35 \mathrm{kGy}$. Before implantation and cementing of the polyethylene component, all synthetic scapula models were sectioned to approximately $6-\mathrm{cm}$ segments to isolate the synthetic bone surrounding the glenoid and to facilitate attachment to the test apparatus. All specimens were prepared without evidence of scapula fracture or peg penetration.

The implanted glenoid specimens were then potted to the same anatomic level of the glenoid neck in polymethylmethacrylate $\left(\mathrm{Coe}^{\circledR}\right.$ Tray Plastic; GC America, Alsip, IL, USA) and were positioned with the corresponding cobalt-chromium humeral head prosthesis aligned directly perpendicular to the glenoid face and centered along the superoinferior axis of the glenoid. The posterior-augmented and standard glenoid component designs articulate with the same humeral head prosthesis. The humeral head component was seated to the deepest
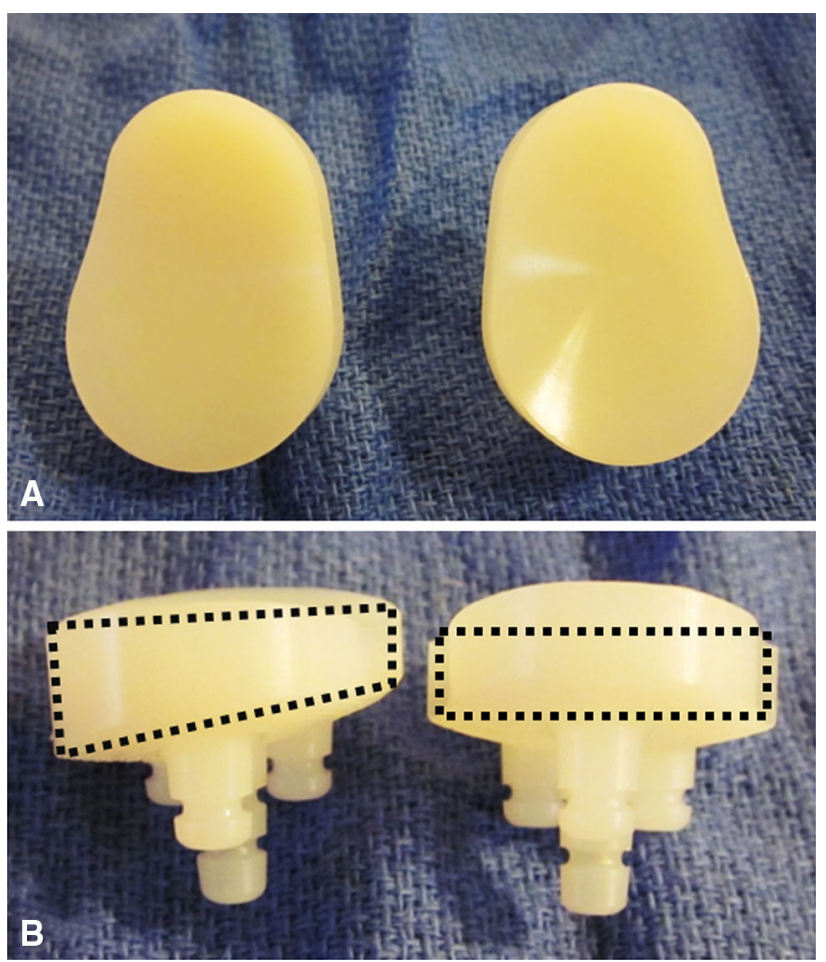

Fig. 1A-B The (A) articular surface of the $8^{\circ}$-posterior augment glenoid component is shown. (B) The view from the inferior edge of the standard glenoid component shows the augmented component (Dashed lines are drawn on the borders of the prosthesis).

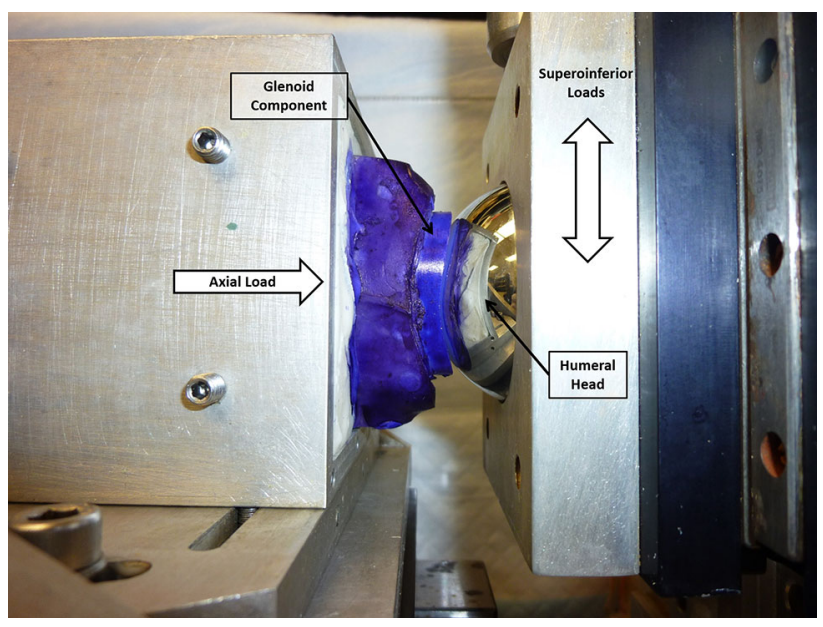

Fig. 2 The testing apparatus used to apply a constant axial load on the glenoid component and cyclic superoinferior loads to the humeral head is shown. Dye was applied to the glenoid component and synthetic bone block to increase contrast between the specimen and the spherical markers (not shown) used for edge displacement analysis.

point on the glenoid face during initial alignment to ensure no posterior subluxation was present.

Testing was performed using a custom apparatus (Fig. 2) attached to an Instron ${ }^{\circledR}$ ElectroPuls ${ }^{\mathrm{TM}}$ E10000 
materials testing machine (Instron Corporation, Norwood, MA, USA). The test protocol (described below) followed the methods proposed in the American Society for Testing and Materials (ASTM) Standard F2028 [2, 3, 33] with the following modifications: (1) subluxation and cyclic tests were performed individually on each specimen, and (2) subluxation testing was done using an axial load of approximately $70 \mathrm{~N}$. The low axial force was selected to avoid damaging the specimens before cyclic testing.

Before cyclic loading, each specimen underwent subluxation translation testing in the superior and inferior directions. While under a constant axial load of $70 \mathrm{~N}$, the humeral head component was displaced at $50 \mathrm{~mm} /$ minute until a peak in shear load was observed. The displacement at peak shear load was defined as the subluxation translation distance, determined individually for each specimen. Axial and shear (superoinferior) loads were applied through an air cylinder and the Instron ${ }^{\circledR}$ test machine actuator, respectively. A calibration curve was generated for the axial load produced by the air cylinder at various pressures. Based on the precision of the pressure gauge (part number KDGF; McDaniel Controls, Luling, LA, USA), the uncertainty in load is approximately $\pm 6.5 \mathrm{~N}$. The shear load was measured directly with a load cell attached to the test machine actuator (10 kN Dynacell; Instron Corporation). The accuracy of this load cell was verified to be better than $\pm 0.5 \%$ down to $1 / 100^{\text {th }}$ capacity. In aggregate, specimens in the eccentric reaming group showed slightly greater superoinferior displacement before subluxation than specimens in the posterior augment group $(3.97 \pm 0.14$ [95\% CI, 3.82-4.12] versus $3.69 \pm 0.25$ [95\% CI, 3.42-3.95 mm]; mean difference, $0.29 \mathrm{~mm}$; $\mathrm{p}=0.036$ ).

After the subluxation tests, specimens were preconditioned under cyclic loading in the superior and inferior directions to $90 \%$ of the previously determined subluxation translations at $0.25 \mathrm{~Hz}$ for 10 cycles while under a constant axial load of 750 N. Specimens then were cyclically loaded for 100,000 cycles at $2 \mathrm{~Hz}$ using $90 \%$ of the predetermined subluxation translations while under a constant axial load of $750 \mathrm{~N}$. This loading protocol represents approximately 25 higher load activities a day for 10 years [3]. In concordance with the ASTM Standard, cyclic loading was performed in the superoinferior direction to reproduce the rocking horse mode of failure in total shoulder arthroplasty [3]. All subluxation, preconditioning, and cyclic testing were performed with the specimens immersed in a circulating heated water bath maintained at $37^{\circ} \mathrm{C}$ for lubrication as dictated by the ASTM standard [3].

The primary outcome measure-glenoid edge displacement-quantifies the motion of the glenoid component in millimeters relative to the synthetic glenoid bone mantle when loaded in the superoinferior direction. These values were determined by imaging 2-mm diameter spherical markers (part \# 9614K71; McMaster-Carr, Los Angeles,
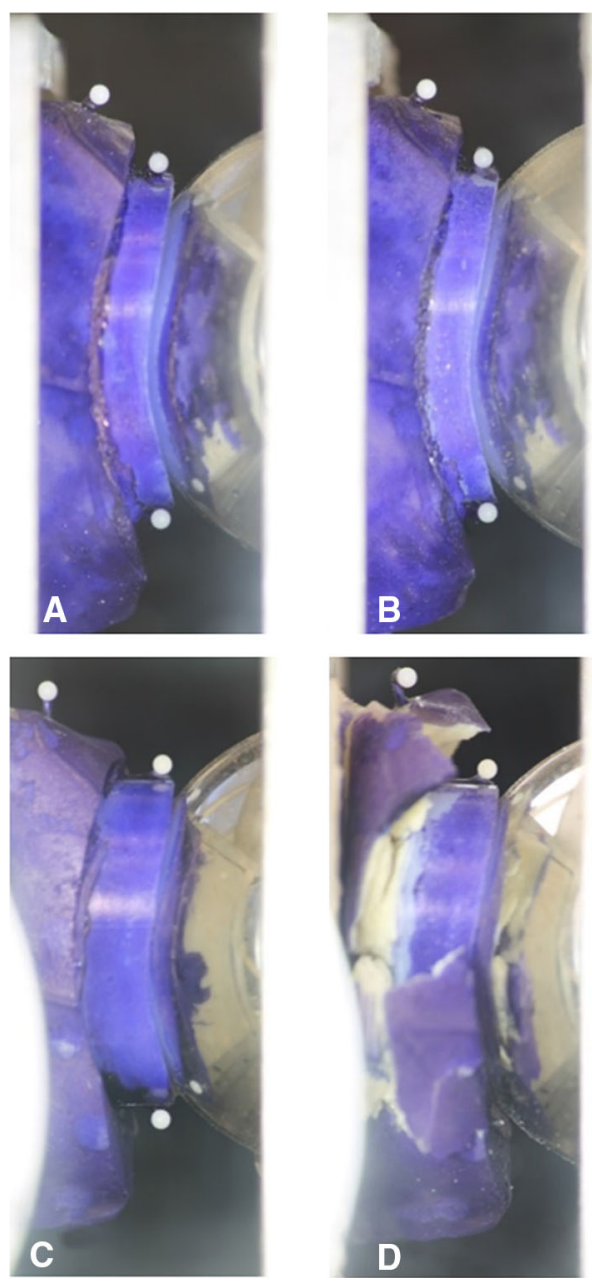

Fig. 3A-D Sample images recorded (A) before and (B) after cyclic testing for the eccentric reaming group, and (C) before and (D) after cyclic testing for the posterior augment group are shown. The spherical markers used to measure edge displacements are attached to the superior and inferior edges of each specimen. Displacement of the superior reference marker and dislodging of the inferior glenoid implant marker can be seen in Illustration D.

CA, USA) secured to $0.9-\mathrm{mm}$ metal wires attached to the superior and inferior edges of the glenoid component and the glenoid neck (Fig. 3). Markers were aligned along the central superoinferior axis of the glenoid. Images for analysis were obtained using a 12.2 Megapixel digital SLR camera (EOS Rebel T3 DSLR with EFS 55-250 mm lens; Canon USA, Melville, NY, USA). Images were recorded with the humeral head positioned at the glenoid origin and then translated to $90 \%$ of the subluxation translation in the superior and inferior directions while under an axial load of 750 N. A custom MATLAB ${ }^{\mathbb{R}}$ program (MathWorks, Natick, MA, USA) was used to analyze the acquired images. The distance, measured perpendicular to the glenoid plane (mediolateral), between the glenoid component markers and the glenoid neck reference marker were determined for each 
image. Edge displacements were defined as the change in distance between the superior and inferior markers and glenoid neck marker with the specimen under edge loading relative to their positions with the humeral head positioned at the origin. "Compressive" edge displacement indicates that the glenoid edge and scapula are pressed together (eg, superior load measuring the superior edge marker), while "distractive" (tensile) edge displacement represents motion leading to separation between the glenoid edge and scapula (eg, superior load measuring the inferior edge marker). Edge displacements were measured following preconditioning and after 100, 1000, 10,000, 50,000, and 100,000 cycles. The average of three individual edge displacement measurements performed at each time was used in the subsequent data analysis. The accuracy and precision of this measurement method are approximately $\pm 0.03 \mathrm{~mm}$ and $\pm 0.02 \mathrm{~mm}$, respectively.

The secondary outcome of glenoid edge load was measured directly during cyclic testing. As the humeral head was translated superiorly or inferiorly, the resistance to motion in Newtons provided by the polyethylene implant in the composite glenoid bone at $90 \%$ of the subluxation translation was recorded.

Testing was terminated and defined as catastrophic failure before 100,000 cycles when the extent of glenoid subsidence (defined as the displacement of the polyethylene glenoid component into the synthetic bone, perpendicular to the glenoid plane) resulted in loosening or destruction of the markers used for edge displacement measurements via comminution of the underlying synthetic scapula surrounding the glenoid component. For all specimens that had catastrophic failure, edge displacement measurements were attempted but were physically unable to be attained as the magnitude of glenoid component instability under axial load caused fragmentation of the surrounding synthetic bone and dislodging of the spherical markers. Consequently, edge displacement and load calculations were determined only for specimens that survived testing to each time. Implant subsidence was measured by calculating the difference in pre- and posttesting images of the mediolateral distance between the glenoid component marker and a stationary point on the testing frame using ImageJ (http://imagej.nih.gov/ij/; National Institutes of Health, Bethesda, MD, USA) with the specimen under a compressive load of $750 \mathrm{~N}$.

One specimen in the eccentric reaming group sustained catastrophic failure before the study endpoint; however, this occurred far earlier than in all other specimens in either group (before 10,000 cycles). Before cyclic loading, this implant exhibited lower superior edge load and increased edge displacement of the inferior marker compared with other specimens in the same group. During posttest analysis, it was determined that this specimen had insufficient cement mantle along the inferior glenoid edge. Although presumed an outlier, the failed eccentric reaming specimen was included in the final analysis.

Initial pilot testing data indicated that a sample size of six specimens in each group would provide a power of 0.80 to detect a difference in edge displacement of $0.20 \mathrm{~mm}$, assuming a standard deviation of $0.10 \mathrm{~mm}$. Additionally, the number of specimens in each group used in this study was twice the recommended sample size in the ASTM standard [3]. Edge displacements and loads were compared between the eccentric reaming and posterior augment groups at a designated time using t-tests. Additional outcome measures included subluxation translation and posttest subsidence and were compared using a t-test and Mann-Whitney U-test, respectively. For all comparisons, significance was set at $p$ less than 0.05 . Statistical analyses were performed using Microsoft Excel (Microsoft Corporation, Redmond, WA, USA) and IBM SPSS ${ }^{\mathbb{R}}$ Statistics 22 (IBM Corporation, Armonk, NY, USA). Data are reported as mean $\pm \mathrm{SD}$.

\section{Results}

No differences in glenoid edge displacements were found between the posterior augment and eccentric reaming groups after preconditioning and after cycles 10, 100, 1000, 10,000 , and 50,000. However, at the 100,000 cycle time, surviving specimens in the posterior augment group showed increased displacement of the superior marker $(1.01 \pm 0.02$ [95\% CI, 0.89-1.13] versus $0.83 \pm 0.10$ [95\% CI, 0.72-0.94 mm]; mean difference, $0.18 \mathrm{~mm} ; \mathrm{p}=0.025$ ) and inferior marker $(1.36 \pm 0.05[95 \% \mathrm{CI}, 1.24-1.48]$ versus $1.20 \pm 0.09$ [95\% CI, $1.09-1.32 \mathrm{~mm}$ ]; mean difference, $0.16 \mathrm{~mm} ; \mathrm{p}=0.038$ ) during superior edge loading compared with specimens in the eccentric reaming group (Fig. 4A). Similarly, specimens in the posterior augment group showed greater displacement of the superior marker during inferior loading compared with specimens in the eccentric reaming group $(1.44 \pm 0.06$ [95\% CI, 1.28-1.59] versus $1.16 \pm 0.11$ [95\% CI, $1.02-1.30 \mathrm{~mm}$ ]; mean difference, $0.28 \mathrm{~mm} ; \mathrm{p}=0.009$ ), whereas no difference was seen for the inferior marker $(0.93 \pm 0.15[95 \% \mathrm{CI}$, $0.56-1.29$ ] versus $0.78 \pm 0.06$ [95\% CI, $0.70-0.85 \mathrm{~mm}$ ]; mean difference, $0.15 \mathrm{~mm} ; \mathrm{p}=0.079$ ) (Fig. 4B). Overall, superior and inferior glenoid implant edge displacement increased as the cycle count increased for specimens in the posterior augment and eccentric reaming groups, indicating progressive implant loosening with time.

No differences were found for superior and inferior edge load measurements between surviving specimens of the posterior augment and eccentric reaming groups at any of the designated times during cyclic loading. Inferior edge 


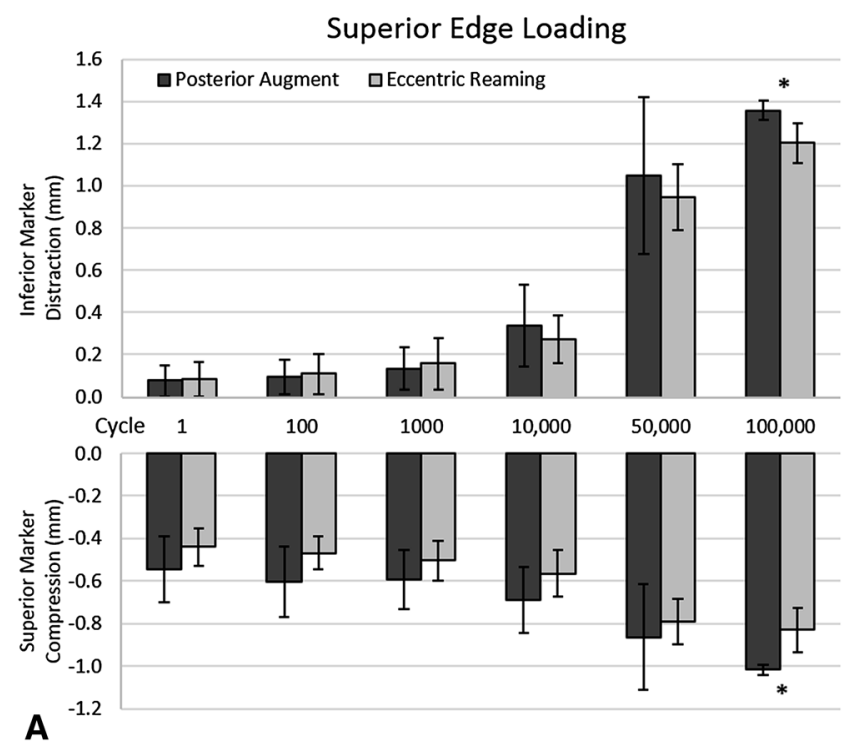

Fig. 4A-B Distractive and compressive edge displacements recorded after specific cycle counts during cyclic testing for (A) superior and (B) inferior edge loading (mean \pm SD) are shown. Distractive displacements were observed in the marker attached to the unloaded edge of the glenoid (eg, inferior marker during superior edge loading),

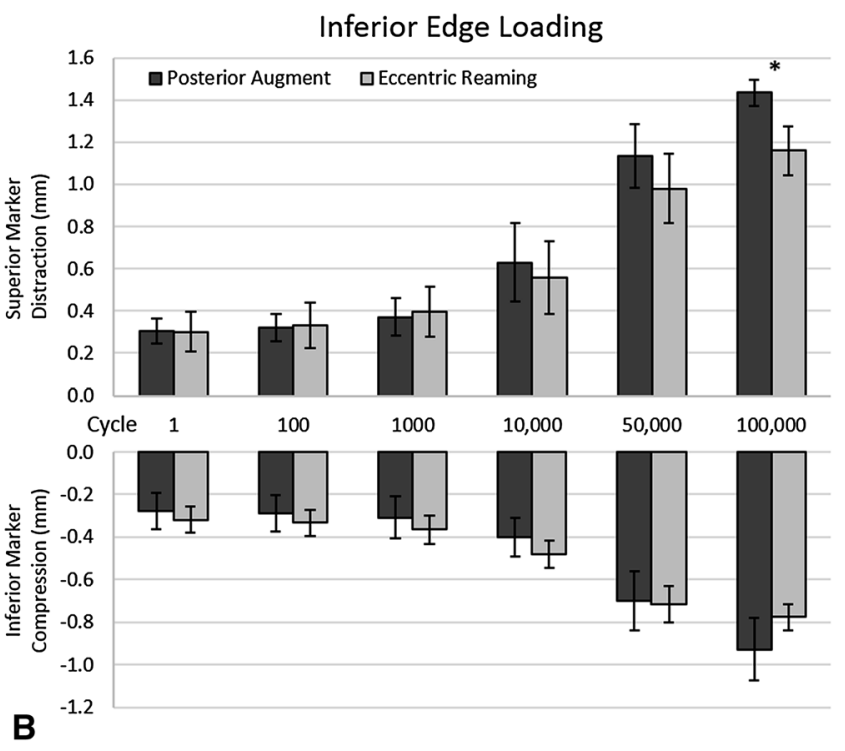

whereas compressive displacements were observed in the marker attached to the loaded edge of the glenoid (eg, superior marker during superior edge loading). * Statistically significant difference between groups $(\mathrm{p}<0.05)$.

Table 1. Specimen survival rate (number of specimens/total sample)

\begin{tabular}{lllllll}
\hline & \multicolumn{2}{l}{ Total cycles } & & & \\
\cline { 2 - 6 } Group & 1 & 100 & 1000 & 10,000 & 50,000 & 100,000 \\
\hline Posterior augment & $6 / 6$ & $6 / 6$ & $6 / 6$ & $6 / 6$ & $5 / 6$ & $3 / 6$ \\
Eccentric reaming & $6 / 6$ & $6 / 6$ & $6 / 6$ & $5 / 6$ & $5 / 6$ & $5 / 6$ \\
\hline
\end{tabular}

load at 100,000 cycles was $186 \pm 45$ (95\% CI, 73-298 N) for the posterior augment group and $242 \pm 27$ (95\% CI, 209-276 N) for the eccentric reaming group (mean difference, $57 \mathrm{~N} ; \mathrm{p}=0.063$ ). Similar to the trend seen with glenoid edge displacement, implant edge load decreased for specimens in the posterior augment and eccentric reaming groups as the cycle count increased, indicating progressive implant instability in the synthetic glenoid.

There was no increased incidence of glenoid vault fracture or implant peg penetration in either group during reaming or implantation. However, as testing progressed, only three of six specimens in the posterior augment group and five of six in the eccentric reaming group were able to achieve the final endpoint of 100,000 cycles without catastrophic failure (Table 1). No differences in implant subsidence were seen between groups when measured at the last available time $(3.3 \pm 3.3$ [95\% CI, -0.21 to 6.78 $\mathrm{mm}]$ posterior augment versus $1.1 \pm 1.6[95 \% \mathrm{CI},-0.58$ to $2.68 \mathrm{~mm}$ ] eccentric reaming; mean difference, $2.24 \mathrm{~mm} ; \mathrm{p}$ $=0.310)$. Regardless of test group, all specimens that failed before 100,000 cycles exhibited at least $4 \mathrm{~mm}$ of glenoid implant subsidence, whereas all that completed 100,000 cycles had less than $0.6 \mathrm{~mm}$ of subsidence.

\section{Discussion}

We evaluated two common techniques to address posterior glenoid wear which is commonly seen during total shoulder arthroplasty for osteoarthritis; eccentric reaming allows placement of a symmetric glenoid component but sacrifices healthy anterior glenoid bone and medializes the joint line, whereas use of a posterior-augmented glenoid component may better preserve glenoid bone architecture by using additional backside polyethylene to restore glenoid version. There is debate regarding the optimal method to correct glenoid version and restore bone stock [22, 36]. Our data suggest that, when subjected to cyclic loading in an in vitro posterior glenoid bone-loss model, eccentric reaming with a standard glenoid component may be biomechanically 
preferable to an angle-backed augmented glenoid component, as measured by decreased implant edge displacement and greater number of specimens reaching the final 100,000 cycle count without catastrophic fracture.

Limitations of this study include the synthetic scapula model used for testing. Initial testing for this study was performed with cadaveric scapulae but was abandoned because of the substantial variability between samples, which had a greater effect on implant stability than prosthesis design. All cadaveric specimens comminuted far earlier than the proposed final time under axial load in the circulating fluid testing environment required by the ASTM standard. The synthetic scapulae we used provided a more homogenous test bed than cadaveric specimens, and they are more anatomically relevant than foam blocks as used in previous studies [8, 23, 33]. Although fatigue testing is not defined for this model, this substrate (fourthgeneration composite bones) has been shown to have similar biomechanical properties to human bone [20, 41, 42] and has been used by others for glenoid prosthesis testing [34]. Despite their homogeneity, the methods used to manufacture the synthetic scapulae result in circularshaped weak zones in the cortical shell. These zones, although consistent in size and location between specimens, may have influenced the failure patterns observed in our study. Despite the presence of these weak zones, both groups of synthetic scapula were subjected to the same glenoid defect and testing protocol, with differences only in specimen preparation and glenoid component type. Although it is difficult to quantify the extent to which the biomechanical model affected the outcomes seen, two findings support the assertion that this synthetic scapula model is still valid in evaluating differences between glenoid component design: (1) excluding the single eccentric reaming specimen that failed owing to insufficient cement, all specimens that had premature failure were treated with the posterior augment implant, and (2) increased glenoid edge displacement was observed in surviving specimens in the posterior augment group during all cycle intervals after 10,000 cycles, compared with the eccentric reaming group. Both of these findings support the assertion that component design was the primary determinant of the findings seen. A final limitation for this study is that in vivo remodeling of subchondral glenoid bone density in osteoarthritis was not modeled and also might affect implant fixation [4, 26, 38]. This limitation is inherent in cadaveric and synthetic test models.

Although the ASTM standard recommends subluxation translation testing be performed on separate samples from those undergoing cyclic loading, we elected to determine these distances individually for each specimen before cyclic loading under a nondestructive axial load to increase group sample sizes. When viewed in aggregate, the humeral head component translated $0.28 \mathrm{~mm}$ more before subluxation for the eccentric reaming group than the posterior augment group. Therefore, specimens in the eccentric reaming group were subjected to greater translation distance per cycle than specimens in the posterior augment group. Despite this difference in subluxation translation, no differences in edge loads were seen at the initial time. Subluxation translation distance has been shown to be dependent on implant geometry alone [3]. We found no statistically significant differences in subsidence between the eccentric reaming and posterior augment groups; however, implant subsidence was approximately threefold larger for specimens in the posterior augment group. Specimens that completed the entire cyclic protocol exhibited very little subsidence $(<0.6 \mathrm{~mm})$, whereas those that exhibited early failure subsided substantially $(>4$ $\mathrm{mm})$. Consequently, the standard deviations for subsidence were large, especially for the posterior augment group which had three specimen failures, and statistical significance was not reached despite the large difference between group means.

Regarding the primary outcome of this study, surviving specimens in the posterior augment group showed greater edge displacement than surviving specimens in the eccentric reaming group at 100,000 cycles, indicating increased component loosening for the posterior augment group. One possible explanation for the increased instability of the augmented component may be the result of its angled backside geometry. When using a standard glenoid component, the axial load is perpendicular to the backside plane of this prosthesis. However, with an angle-backed component, the vector of axial load is oblique to the backside and introduces shear stresses to the implant-bone interface [17]. This may lead to increased micromotion and instability of the prosthesis under cyclic loading. Iannotti et al. [23] compared augmented glenoid component designs in a synthetic bone block model and found a step-cut glenoid component produced decreased anterior glenoid edge liftoff values compared with an angle-backed design, concluding in vitro stability was better with a stepped implant. Analogous findings have been described for knee arthroplasty when evaluating tibial bone loss [7, 30]. Wedge-shaped defects in the tibia introduce destabilizing shear forces and cause decreased stiffness under axial load [7]. The conversion of an oblique wedge defect to a stepcut pattern decreased shear stress and improved implant rigidity by $28 \%$ to $36 \%$. In a study of tibial augments for bone deficiency, $100 \%$ of wedge-shaped constructs failed, whereas none of the step-cut constructs failed under axial load [30]. Clinically, the use of oblique wedge augments for tibial bone deficiency results in $27 \%$ to $46 \%$ incidence of radiolucent line formation 3 to 5 years postoperatively $[6,28-30]$. 
A secondary, indirect measure of glenoid loosening is a reduction in edge loads. As the implant loosens, a load that is applied eccentrically from the center of the glenoid component results in rotation of the glenoid component. This rotation minimizes the effective curvature of the glenoid and results in decreased constraint against superoinferior (shear) loads. Although no differences in edge load were seen between groups, specimens treated with posterior augment and eccentric reaming showed progressively decreasing edge loads as cyclic testing progressed.

Although we found no differences in cortical perforation rates during preparation, the number of specimens that experienced catastrophic implant loosening and failed to achieve 100,000 cycles was greater in the posterior augment group than the eccentric reaming group. Clinical studies using augmented glenoid components to address posterior glenoid wear have shown inconsistent results [9, 30]. Rice et al. [31] reported $86 \%$ of patients treated with a keeled augmented polyethylene glenoid component had satisfactory or excellent results. However, they found this implant did not predictably improve glenohumeral instability. Cil et al. [9] reviewed patients treated with a metalbacked augmented glenoid component and found only a $31 \%$ implant survival rate 7 years postoperatively. Failure was often the result of glenoid component loosening and these implants showed only limited success in correcting subluxation.

In our study, we found increased implant edge displacement and greater incidence of catastrophic implant loosening during cyclic testing in specimens prepared with an angle-backed posterior-augmented glenoid component compared with a standard glenoid component after eccentric reaming. Angle-backed posterior augment glenoid components may introduce shear stress across the glenoidbone interface during axial loading, potentially compromising stability and leading to early failure resulting from loosening. Additionally, the differences in amount and morphologic features of remaining glenoid bone stock might influence stability of the prosthesis. Additional in vitro studies of subtypes of augmented implant designs and long-term clinical followup, including physician reporting and arthroplasty registries, are recommended for further evaluation of this component design.

Acknowledgments We thank Exactech, Inc (Gainesville, FL, USA) for donation of implants and instruments.

\section{References}

1. Adams JE, Sperling JW, Hoskin TL, Melton LJ 3rd, Cofield RH. Shoulder arthroplasty in Olmsted County, Minnesota, 1976-2000: a population-based study. J Shoulder Elbow Surg. 2006;15:50-55.
2. Anglin C, Wyss UP, Pichora DR. Mechanical testing of shoulder prostheses and recommendations for glenoid design. $J$ Shoulder Elbow Surg. 2000;9:323-331.

3. ASTM F2028-08, 2008. "Standard Test Methods for Dynamic Evaluation of Glenoid Loosening or Disassociation". West Conshohocken, PA: ASTM International; 2008. Available at: http//:www.astm.org. Accessed July 14, 2015.

4. Beuckelaers E, Jacxsens M, Van Tongel A, De Wilde LF. Threedimensional computed tomography scan evaluation of the pattern of erosion in type B glenoids. J Shoulder Elbow Surg. 2014; 23:109-116.

5. Bohsali KI, Wirth MA, Rockwood CA Jr. Complications of total shoulder arthroplasty. $J$ Bone Joint Surg Am. 2006; 88:2279-2292.

6. Brand MG, Daley RJ, Ewald FC, Scott RD. Tibial tray augmentation with modular metal wedges for tibial bone stock deficiency. Clin Orthop Relat Res. 1989;248:71-79.

7. Chen F, Krackow KA. Management of tibial defects in total knee arthroplasty: a biomechanical study. Clin Orthop Relat Res. 1994; 305:249-257.

8. Chong AC, Friis EA, Ballard GP, Czuwala PJ, Cooke FW. Fatigue performance of composite analogue femur constructs under high activity loading. Ann Biomed Eng. 2007; 35:1196-1205.

9. Cil A, Sperling JW, Cofield RH. Nonstandard glenoid components for bone deficiencies in shoulder arthroplasty. J Shoulder Elbow Surg. 2014;23:e149-157.

10. Clavert P, Millett PJ, Warner JJ. Glenoid resurfacing: what are the limits to asymmetric reaming for posterior erosion? $J$ Shoulder Elbow Surg. 2007;16:843-848.

11. Collins D, Tencer A, Sidles J, Matsen F 3rd. Edge displacement and deformation of glenoid components in response to eccentric loading: the effect of preparation of the glenoid bone. $J$ Bone Joint Surg Am. 1992;74:501-507.

12. DeFrances CJ, Lucas CA, Buie VC, Golosinskiy A. 2006 National Hospital Discharge Survey. Natl Health Stat Report. 2008;30:1-20.

13. Edwards TB, Labriola JE, Stanley RJ, O'Connor DP, Elkousy HA, Gartsman GM. Radiographic comparison of pegged and keeled glenoid components using modern cementing techniques: a prospective randomized study. J Shoulder Elbow Surg. 2010; 19:251-257.

14. Farron A, Terrier A, Büchler P. Risks of loosening of a prosthetic glenoid implanted in retroversion. J Shoulder Elbow Surg. 2006; 15:521-526.

15. Fox TJ, Foruria AM, Klika BJ, Sperling JW, Schleck CD, Cofield RH. Radiographic survival in total shoulder arthroplasty. $J$ Shoulder Elbow Surg. 2013;22:1221-1227.

16. Gillespie R, Lyons R, Lazarus M. Eccentric reaming in total shoulder arthroplasty: a cadaveric study. Orthopedics. 2009; 32:21.

17. Giori NJ, Beaupré GS, Carter DR. The influence of fixation peg design on the shear stability of prosthetic implants. J Orthop Res. 1990;8:892-898.

18. Gregory T, Hansen U, Taillieu F, Baring T, Brassart N, Mutchler C, Amis A, Augereau B, Emery R. Glenoid loosening after total shoulder arthroplasty: an in vitro CT-scan study. J Orthop Res. 2009;27:1589-1595.

19. Habermeyer P, Magosch P, Lichtenberg S. Recentering the humeral head for glenoid deficiency in total shoulder arthroplasty. Clin Orthop Relat Res. 2007;457:124-132.

20. Heiner AD. Structural properties of fourth-generation composite femurs and tibias. J Biomech. 2008;41:3282-3284.

21. Hill JM, Norris TR. Long-term results of total shoulder arthroplasty following bone-grafting of the glenoid. J Bone Joint Surg Am. 2001;83:877-883. 
22. Hsu JE, Ricchetti ET, Huffman GR, Iannotti JP, Glaser DL. Addressing glenoid bone deficiency and asymmetric posterior erosion in shoulder arthroplasty. J Shoulder Elbow Surg. 2013;22:1298-1308.

23. Iannotti JP, Lappin KE, Klotz CL, Reber EW, Swope SW. Liftoff resistance of augmented glenoid components during cyclic fatigue loading in the posterior-superior direction. J Shoulder Elbow Surg. 2013;22:1530-1536.

24. Jones RB. Addressing glenoid erosion in anatomic total shoulder arthroplasty. Bull Hosp Jt Dis (2013). 2013;71(suppl 2):S46-50.

25. Kim SH, Wise BL, Zhang Y, Szabo RM. Increasing incidence of shoulder arthroplasty in the United States. J Bone Joint Surg Am. 2011;93:2249-2254.

26. Knowles NK, Athwal GS, Keener JD, Ferreira LM. Regional bone density variations in osteoarthritic glenoids: a comparison of symmetric to asymmetric (type B2) erosion patterns. J Shoulder Elbow Surg. 2015;24:425-432.

27. Norris TR, Iannotti JP. Functional outcome after shoulder arthroplasty for primary osteoarthritis: a multicenter study. $J$ Shoulder Elbow Surg. 2002;11:130-135.

28. Pagnano MW, Trousdale RT, Rand JA. Tibial wedge augmentation for bone deficiency in total knee arthroplasty: a followup study. Clin Orthop Relat Res. 1995;321:151-155.

29. Rand JA. Bone deficiency in total knee arthroplasty: use of metal wedge augmentation. Clin Orthop Relat Res. 1991;271:63-71.

30. Rand JA. Modular augments in revision total knee arthroplasty. Orthop Clin North Am. 1998;29:347-353.

31. Rice RS, Sperling JW, Miletti J, Schleck C, Cofield RH. Augmented glenoid component for bone deficiency in shoulder arthroplasty. Clin Orthop Relat Res. 2008;466:579-583.

32. Rispoli DM, Sperling JW, Athwal GS, Wenger DE, Cofield RH. Projection of the glenoid center point within the glenoid vault. Clin Orthop Relat Res. 2008;466:573-578.

33. Roche C, Angibaud L, Flurin PH, Wright T, Zuckerman J. Glenoid loosening in response to dynamic multi-axis eccentric loading: a comparison between keeled and pegged designs with an equivalent radial mismatch. Bull Hosp Jt Dis. 2006;63: 88-92.

34. Roche CP, Stroud NJ, Martin BL, Steiler CA, Flurin PH, Wright TW, Zuckerman JD, Dipaola MJ. Achieving fixation in glenoids with superior wear using reverse shoulder arthroplasty. J Shoulder Elbow Surg. 2013;22:1695-1701.

35. Sabesan V, Callanan M, Sharma V, Iannotti JP. Correction of acquired glenoid bone loss in osteoarthritis with a standard versus an augmented glenoid component. J Shoulder Elbow Surg. 2014;23:964-973.

36. Sears BW, Johnston PS, Ramsey ML, Williams GR. Glenoid bone loss in primary total shoulder arthroplasty: evaluation and management. J Am Acad Orthop Surg. 2012;20:604-613.

37. Shapiro TA, McGarry MH, Gupta R, Lee YS, Lee TQ. Biomechanical effects of glenoid retroversion in total shoulder arthroplasty. J Shoulder Elbow Surg. 2007;16(3 suppl):S90-95.

38. Simon P, Gupta A, Pappou I, Hussey MM, Santoni BG, Inoue N, Frankle MA. Glenoid subchondral bone density distribution in male total shoulder arthroplasty subjects with eccentric and concentric wear. J Shoulder Elbow Surg. 2015;24:416-424.

39. Steinmann SP, Cofield RH. Bone grafting for glenoid deficiency in total shoulder replacement. $J$ Shoulder Elbow Surg. 2000;9:361-367.

40. Walch G, Badet R, Boulahia A, Khoury A. Morphologic study of the glenoid in primary glenohumeral osteoarthritis. J Arthroplasty. 1999;14:756-760.

41. Zdero R, Elfallah K, Olsen M, Schemitsch EH. Cortical screw purchase in synthetic and human femurs. $J$ Biomech Eng. 2009;131:094503.

42. Zdero R, Olsen M, Bougherara H, Schemitsch EH. Cancellous bone screw purchase: a comparison of synthetic femurs, human femurs, and finite element analysis. Proc Inst Mech Eng $H$. 2008;222:1175-1183. 